\title{
Diagnóstico E TRATAMENTO DE TUMORES CARCINÓIDES DO TRATO DIGESTIVO
}

\author{
Luis César Fernandes*, Luiz Pucca, Delcio Matos \\ Trabalho realizado na disciplina de Gastroenterologia Cirúrgica do Departamento \\ de Cirurgia da Universidade Federal de SãoPaulo(UNIFESP-EPM).
}

RESUMO - Os autores verificam as características dos tumores carcinóides de acordo com sua localização no trato digestivo. Apesar de sua baixa velocidade de crescimento e de metastatização, tais neoplasias são malignas e devem ser submetidas a ressecção cirúrgica. Casos avançados com metástases são tratados com derivados da somatostatina, com paliação dos

\section{INTRODUÇÃO}

Os tumores carcinóides são neoplasias do sistema celular neuroendócrino difuso. Otermo "tumor neuroendócrino" tem sido proposto para a referida entidade $1,2,3,4$.

É certo o envolvimento de fatores genéticos na etiologia. É provável que a deleção do gen supressor PLCß3 provoque descontrole de crescimento de células neuro-endócrinas, com distorção do processo de apoptose $e^{5,6}$ e desenvolvimento de neoplasias.

A incidência de tumores carcinóides oscila em 0,7 casos por 100.000 habitantes ${ }^{7,8}$. A incidência real deve ser maior, considerando-se um achado mais freqüente observado em necrópsias? ${ }^{9}$ Certo número de lesões incipientes não deve ultrapassar estágios iniciais no decorrer da existência.

Os doentes têm média etária de 50 anos quando se efetua o diagnóstico da neoplasia ${ }^{7,10}$. As mais freqüentes sedes de tumores carcinóides são o trato gastrintestinal

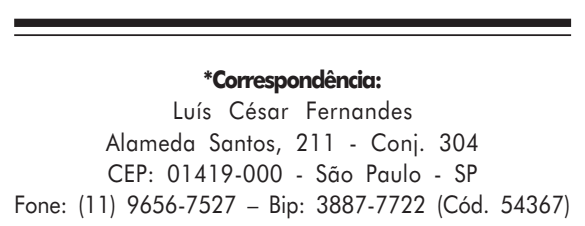

(73,7\%) e o sistema respiratório $(25,1 \%)$. No aparelho digestivo, os principais órgãos afetados são intestino delgado, apêndice cecale reto (Tabelal). Têm ocorrido mais diagnósticos em virtude de um melhor conhecimento do processo neoplásico e dos aperfeiçoamentos obtidos nos métodos de investigação disponíveis; contudo, aparentemente, a incidência tem se elevado nas últimas duas décadas".

\section{Classificação e fisiopatologia}

Os tumores carcinóides têm sido classificados de acordo com sua formação embriológica, se originários do intestino anterior, médio ou posterior ${ }^{12}$.

Os tumores de origem embrionária no intestino anterior acometem, na fase adulta, o trato respiratório e o timo. Os originários no intestino médio são os de maior freqüência, manifestando-se em jejuno, íleo e cólon direito e sendo os maiores produtores de serotonina. Lesões inerentes ao intestino posterior embrionário acometem o cólon esquerdo e o reto na fase adulta ${ }^{9}$, raramente originando a síndrome carcinóide, por produzirem pouca serotonina ${ }^{12}$.

Os tumores carcinóides podem ter diferentes perfis de produção hormonal de acordo com seu sítio de origem. Podem produzir serotonina, ACTH, calicreína, histamina, catecolaminas, prostaglandinas, gastrina e insulina, gerando diversas reações orgânicas e originando diferentes quadros clínicos 4 . São exemplos de 
Fernandes LCetal.

reações: serotonina - ondas de calor, ACTH - rubor facial, calicreína - diarréia. Possuem, geralmente, baixa velocidade de crescimento e de metastatização.

\section{Diagnóstico geral}

A sintomatologia é diversas vezes frustra, variando de acordo com o perfil de produção endócrina e com a localização do tumor, o que gera dificuldades para que se efetue um diagnóstico baseado no quadro clínico. É, mesmo, rara a hipótese pré-operatória de tumor carcinóide. Normalmente, o diagnóstico se faz por análise histológica de lesões de natureza a esclarecer, no período pré ou no pós-operatório. Por vezes, neoplasias acidentalmente encontradas e ressecadas apresentam surpreendentemente 0 diagnóstico ao exame anatomopatológico. A característica síndrome carcinóide, com fogachos, rubor facial e diarréia, é de rara ocorrência, incidindo em 3\% a 5\% dos pacientes, geralmente já possuidores de neoplasias em estágio avançado de desenvolvimento e de disseminação?

A elevação urinária do ácido 5-hidroxiindolacético (5-HIAA) constitui ainda método útil para o diagnóstico, ${ }^{9}$. Vários exames de imagem podem ser usados no diagnóstico, localização e estadiamento de tumores carcinóides, tais como: radiografia de abdômen e exames contrastados ${ }^{14}$, ultrasonografia abdominal (US), tomografia computadorizada $(\mathrm{CT})^{15,16}$, ultra-sonografia endoscópica e endorretal (USER) e ressonância nuclear magnética (RNM), com valores diferentes de acurácia, sempre inferiores a 50\% 17,18. Exames mais específicos são baseados em cintilografia, com utilização de isótopos ligados a derivados da somatostatina, alcançando acurácia superior a $80 \% \%^{14,19,20}$.

Nos exames anatomopatológicos inconclusivos o emprego de marcadores imunohistoquímicos, tais como a cromogranina-Ae a sinaptofisina, possibilitam a definição do quadro ${ }^{6}$.

\section{Tratamento cirúrgico geral}

Lesões carcinóides são neoplasias malignas e como tais devem ser tratadas. A cirurgia é prioritária, com complementação por métodos adjuvantes, se necessário. As táticas cirúrgicas variam de acordo com o órgão sede e com as características da doença para determinada localização.

\section{Esôfago}

Sítio extremamente raro, a incidência neste local eqüivale a $0,04 \%$ dos casos de tumor carcinóide, tendo sido descritos somente três casos em levantamento de 8305 pacientes portadores desse tipo de neoplasia nos EEUU"'. Devido a sua extremamente baixa freqüência não há relatos ou propostas de padronização de procedimentos. A conduta clínicocirúrgica deve ser guiada pelas diretrizes gerais para neoplasias malignas nesse órgão.

\section{Estômago}

A sintomatologia das lesões carcinóides gástricas éinespecífica. Geralmente, ocorre dispepsia que pode ser creditada a outras alterações possíveis, sendo as lesões carcinóides diagnosticadas à investigação endoscópica desses casos. Há três tipos de tumor gástricos carcinóides. O Tipo I, mais comum, está associado com gastrite crônica atrófica. OpHneutro do estômago estimula a constante secreção de gastrina e a persistente hipergastrinemia resulta no desenvolvimento de múltiplos carcinóides, geralmente benignos, situados nas regiões de corpo e fundo gástricos ${ }^{21}$. Os de Tipo II estão associados com síndrome de Zollinger-Ellison. Embora sejam relativamente benignos, têm potencial maior de metastatização que os do Tipo I. Os do Tipo III, sem associação com outras entidades ou concentração de gastrina, são esporádicos e os mais agressivos ${ }^{22,23,24}$.

A maioria dos tumores gástricos não cursa com disseminação metastática, possi- velmente pelo atual diagnóstico precoce efetuado ${ }^{21}$. Ocasionalmente, porém, sintomas decorrentes de metastatização para o território hepático podem ser os primeiros a ocorrer. Endoscopia com múltiplas biópsias e remoção de lesões polipóides constituiem opção adequada ${ }^{25}$. Por vezes, os carcinóides do estômago podem ser sésseis e de base larga, ocasiões para as quais tem sido descrita a ressecção mucosa endoscópica ("strip biopsy") para o tratamento local, em lesões de diâmetro inferior a $1 \mathrm{~cm}$, com remota possibilidade de metastatização linfonodal ${ }^{26}$.

Para pacientes com tumores maiores que $2 \mathrm{~cm}$ de diâmetro e produção de gastrina, antrectomia com ressecção da lesão e linfadenectomia regional é a estratégia de adequaçãa ${ }^{25}$. Pacientes com tumores de diâmetros superiores a $1 \mathrm{~cm}$ sem hipergastrinemia (Tipo III) devem ser submetidos a gastrectomia total com linfadenectomia regional ${ }^{27}$.

\section{Duodeno}

Os carcinóides desta localização correspondem a $2 \%$ dos tumores neuroendócrinos do trato gastrintestina|l| ${ }^{2 !}$ Os mais freqüentes são os gastrinomas. Geralmente, situam-se na la ou $2^{a}$ porções duodenais, sendo de diâmetro inferior a I cm. Há forte tendência à disseminação linfonodal, que ocorre em 30 a $70 \%$ dos $\operatorname{casos}^{28}$. Geralmente, as metástases são maiores que o tumor primário, o qual por vezes é de difícil detecção à cirurgia ${ }^{21}$.

O tratamento curativo somente pode ser proporcionado cirurgicamente, variando desde ressecção local até pancreaticoduodenectomia, dependendo da dimensão do tumor, da idade e condições clínicas do paciente. Lesões de diminutas dimensões confinadas à submucosa podem ser estadiadas e ressecadas pelo auxílio de ecoendoscopia ${ }^{29,30}$. Nas lesões pequenas e localizadas propõe-se longa duodenotomia e eversão mucosa para palpação, identificação e exérese da lesão. Grandes lesões, raras, reque- 
rem pancreaticoduodenectomia ${ }^{21}$. Em tumores irressecáveis, as cirurgias de derivação são preconizadas.

\section{Pâncreas}

Raros são os tumores neuroendócrinos do pâncreas. A sintomatologia é variável e o diagnóstico pode ser feito com métodos de imagem (ultra-sonografia abdominal, tomografia computadorizada e ressonância nuclear magnética), cintilografia com análogos da somatostatina e ecoendoscopia ${ }^{31}$. Devem ser excisados cirurgicamente ${ }^{21}$, com técnica variando conforme localização pancreática, as dimensões do tumor e a possível disseminação.

\section{Intestino delgado}

Geralmente, os tumores carcinóides desta localização são muito pequenos e incidem no ileo terminal ${ }^{25}$. As lesões primárias têm diâmetro entre 1 e $2 \mathrm{~cm}$, enquanto que as lesões secundárias são maiores e, por vezes, passíveis de diagnóstico antes da descoberta da lesão primária ${ }^{32}$. O envolvimento de linfonodos ocorre mesmo para lesões menores que I cm, com elevada freqüência. Há comprometimento linfonodal em 20\% a 30\% dos pacientes com tumores menores que I $\mathrm{cm}^{33} \mathrm{e} \mathrm{em} 85 \%$ dos pacientes com lesões de $2 \mathrm{~cm}$ de diâmetro ou mais ${ }^{34}$.

A maioria dos pacientes é submetida a tratamento cirúrgico por sinais e sintomas de obstrução intestinal. De modo interessante, a obstrução não é usualmente causada pelo tumor, mas sim por reação atingindo o mesentério originando acentuada retração e fibrose ${ }^{32}$.

O tratamento consiste na ampla ressecção da lesão e extensa excisão do território de drenagem linfática regional, mesmo para pequenas lesões ${ }^{32}$.

O exame intra-operatório meticuloso é primordial, pois em $20 \%$ a $40 \%$ dos casos alesão é multicêntrica, porvezes com aspecto amarelado visível pela face serosa da alça intestinal $^{32}$.

\section{Cólon}

A maioria dos carcinóides cólicos são encontrados no cólon direito.

O diagnóstico se inicia pelo advento de dor abdominal em cólica e perda de peso, quando há sintomas, levando à realização de exames complementares ${ }^{32}$. Por vezes, o diagnóstico é realizado durante exame endoscópico motivado por sintomas não decorrentes da lesão neoplásica. A colonoscopia, com realização de biópsia e de exame histológico da lesão, estabelece o diagnóstico. Posteriormente, exames de imagem possibilitam determinar o grau de disseminação da neoplasia e a conduta terapêutica adequada.

Detectados pela colonoscopia, tumores com diâmetro inferior a $2 \mathrm{~cm}$ e pediculados podem ser removidos por polipectomia ${ }^{25}$. Nos tumores cólicos de maiores dimensões deve-se empreender a ressecção padrão do segmento afetado, incluindo o território de drenagem linfática regiona ${ }^{32}$.

\section{Apêndice}

Nos tumores apendiculares o diagnóstico é geralmente efetuado após excisão do órgão, em quadro sugestivo de apendicite ou por detecção de alteração no apêndice durante intervenção cirúrgica abdominal motivada por doença em outro órgão intracavitário. O estudo histopatológico demonstra a natureza real da lesão e define a conduta subseqüente a seguir ${ }^{22,33}$. A maioria dos tumores ocorre no apêndice distal e não há dificuldades em seu tratamento ${ }^{25}$.

Em tumores menores e desprovidos de caráter invasivo, a simples apendicectomia serásuficiente para o tratamento definitivo da lesão ${ }^{32}$. Porém, há situações clínicas em que se torna imperiosa avaliação para decisão sobre a necessidade ou não de realização de intervenção cirúrgica maior. São precisas indicações para reintervenção e realização de hemicolectomia direita com excisão da cadeia de drenagem linfática local: tumores que invadem a parede cecal; lesões maiores que 2 cm de diâmetro; produção de mucina; invasão de linfáticos, serosa ou mesoapêndice e pleomorfismo celular com elevado índice de mitoses ${ }^{35}$.

\section{Reto}

Aproximadamente $50 \%$ dos tumores carcinóides do reto provocam sintomas, principalmente sangramento, constipação, dor e prurido ana| ${ }^{32}$. Feito o diagnóstico de lesão carcinóide, o US endorretal possibilitará avaliação do grau de infiltração local ${ }^{18}$. A CT de pelve e de abdômen, a cintilografia e outros exames de imagem permitirão verificação do grau de disseminação local e à distância, auxiliando na definição do tratamento a ser estabelecido ${ }^{36}$.

É fundamental a verificação da profundidade de invasão na parede do reto. Se não houver invasão das camadas musculares ese a lesão for inferior a I cm de diâmetro, a ressecção endoscópica ${ }^{37}$ ou a ressecção local, esta preferencial sempre que factível ${ }^{32}$, são métodos habitualmente suficientes. No entanto, preconiza-se excisão cirúrgica radical, por ressecção anterior ou por amputação abdominoperineal do reto, de todos os tumores carcinóides retais em que haja invasão da muscular própria ${ }^{38}$, dimensões maiores que I cm de diâmetro, elevada atividade mitótica ou ulceração de mucosa ${ }^{32,39}$.

Há que se verificar que, em muitas situações, a lesão retal é interpretada inicialmente como pólipo de natureza benigna, o qual é submetido à excisão endoscópica, sendo efetuado o diagnóstico de tumor neuroendócrino somente ao exame anatomopatológico posteriormente efetuado ${ }^{37}$. Os mesmos critérios descritos inicialmente devem ser seguidos no tratamento de tais lesões, ampliando-se a ressecção sempre que se tornar necessário.

Deve-se considerar que, talvez, ressecções cirúrgicas ampliadas para tumores carcinóides retais muito raramente devam 
Fernandes LCetal.

ser indicadas. Pequenas lesões são eficientemente tratadas por ressecções locais. Lesões maiores geralmente já apresentam metastatização, sendo que amputações retais por via abdominal ou abdominoperineal em tumores com disseminação não melhoram a expectativa de vida dos pacientes. $O$ tratamento deve se restringir, nesses casos, também a ressecção local da lesão, para paliação dos sintomas regionais ${ }^{32,39,40}$, com tratamento adjuvante a seguir.

\section{Fígado}

Tumores neuroendócrinos comumente metastatizam para o fígado, sendo no contexto de neoplasias secundárias que os carcinóides devem ser abordados em situação hepática ${ }^{41}$.

Em pacientes com metástases restritas ao território hepático, aos exames de imageme de cintilografia ${ }^{42}$, é possível a realização de ressecções hepáticas pretensamente curativas ${ }^{43,44}$. A exérese de metástases hepáticas com radicalidade pode elevar oíndice de sobrevida dos pacientes em cinco anos de $29 \%$ para $73 \%{ }^{41}$. Ressecções paliativas de metástases hepáticas, com o intuito de amenizar os sintomas da síndrome carcinóide por redução da massa tumoral, somente estão indicadas se mais de $90 \%$ do tecido neoplásico puder ser excisado?. A criocirurgia tem sido proposta para o tratamento de metástases hepáticas irressecáveis ${ }^{45,46}$. Mesmo o transplante hepático para tratamento de tumor carcinóide metastático pode ser aventado, em casos selecionados, se houver perspectiva de radicalidade cirúrgica e de curabilidade ${ }^{47,48}$.

\section{Tratamento coadjuvante}

Utilizado em casos de doença disseminada, pode visar o tratamento de metástases hepáticas, de múltiplas metástases em diversos sítios do organismo ou a remissão de sintomas que comprometam a qualidade de vida do paciente.

Diversos métodos terapêuticos adju- vantes têm sido empregados e testados, tais como: radioterapia ${ }^{49}$, quimioterapia (5-fluorouracil, dacarbazina, epirubicina $a^{50}$, adriamicin $^{33}$ e outros compostos) e uso de interferon- $\alpha^{51}$, com relativa melhora na sintomatologia não correspondente a melhores efeitos antitumorais s2 $^{2}$

A utilização de derivados da somatostatina tem tido destacada importância no tratamento clínico do tumor carcinóide, pelo controle da sintomatologia de síndrome carcinóide ${ }^{53,54}$ e, inclusive, indução de apoptose e de regressão tumoral quando utilizados em alta dosagem ${ }^{55}$. Os derivados mais utilizados atualmente têm sido o octreotide, doses de 0,05 a 0,5 mg SC três vezes ao dia $\mathrm{a}^{56}$, e olanreotide, I $\mathrm{mg}^{57}$ ou $5 \mathrm{mg}{ }^{58} \mathrm{SC}$ três vezes ao dia. São inconvenientes a freqüência das administrações injetáveis, as reações observadas nos locais de aplicação e o custo do tratamento para os pacientes. De recente lançamento, encontra-se o octreotide injetável em preparado de depósito para liberação prolongada (LAR), com uma aplicação mensal IM de 20 mg $\mathrm{g}^{25}$, com melhor adesão do paciente ao tratamento, porém com elevado custo de manutenção.

No tratamento de metástases hepáticas irressecáveis têm sido empregadas: desarterialização (cirúrgica ou por embolização por arteriografia, 59,60 ) e a quimioembolizaçãa $0^{36,61}$. Deve-se lembrar que a irrigação dos tumores carcinóides no figado é predominantemente arterial; a oclusão desses vasos em território hepático geraimportante regressão das lesões.

\section{Prognóstico}

O tumor carcinóide permanece com lento crescimento por prolongados períodos de tempo. Porém, isto, absolutamente, não deve levar a posição indolente na assistênciaterapêutica. Asobrevida dos pacientes com doença disseminada por ocasião do diagnóstico e sem tratamento era de $18 \%$ em cinco anos ${ }^{62}$. Os tratados com análogos da somatostatina em idênticas condições clínicas têm apresentado índices de 67\% de sobrevida em cinco anos, com sobrevivência média total de 12 anos após o diagnóstico de doença metastática ${ }^{19}$. Portanto, mesmo em casos com disseminação, o tratamento deve ser tentado, preferencialmente com derivados e análogos da somatostatina.

Apesar disso, tumores com origem no intestino médio embrionário induzem a menores índices de sobrevida que os originários no intestino posterior ${ }^{63}$.

Em relação às dimensões tumorais, as lesões maiores que $2 \mathrm{~cm}$ apresentam potencial de metastatização maior ${ }^{10,64}$. Entretanto, mesmo lesões primárias inferiores a $2 \mathrm{~cm}$ podem incorrer com a existência de disseminaçãotumoral a distância ${ }^{65}$. Aexistência de metástases linfonodais deve sempre ser investigada.

A sobrevida geral atual para pacientes tratados com tumores carcinóides de todas as localizações oscila por volta de $50 \%$ em cinco anos. Oscila em 85,9\% para lesões no apêndice, 41,6\% no cólon e 72,2\% no reto". É possível que o diagnóstico precoce das lesões apendiculares e retais justifique melhores índices de sobrevida para processos de tais localizações ${ }^{66}$.

É freqüente a coexistência de tumores carcinóides com outras neoplasias, chegando a $17 \%$, segundo algumas estatísticas. Os principais órgãos sedes de neoplasias associadas são colo uterino e cólon, o que impõe a necessidade de pesquisa de tumores associados ${ }^{11,66,67}$.

\section{SUMMARY}

\section{DIAGNOSIS AND TREATMENT FOR CARCI-} NOID TUMORS IN THE DIGESTIVE TRACT.

The authors analyzed the characteristics of carcinoid tumors according to their site in the digestive tract. Despite theirlow speed of growing and metastasis process, such neoplasias are malignant and must be submitted to surgical resection. Advanced cases with 
metastases are treated with somatostatin derivatives, lessening symptoms, causing occasional regression of lesions and presenting an extended survival. Appendicular and rectal lesions have a better prognosis maybe due to the early diagnosis performed. [Rev Assoc Med Bras 2002; 48(1): 87-92]

\section{KEYwORDS: Carcinoid tumor.Gastrointestinal} neoplasms. Neuroendocrine tumors. Digestive system neoplasms.

\section{REFERÊNCIAS}

I. Oberndorfer S. Uber die "kleinen Dunndarm Carcinome". Verh Dtsch Ges Pathol 1907; | $1: 1 \mid 3-6$.

2. Masson P. Carcinoids (argentafin-cell tumors) and nerve hyperplasia of appendicular mucosa. Am J Pathol 1928; 4:81-212.

3. Lembech F. 5-Hydroxytryptamine in carcinoid tumor. Nature 1953; 172: 910-1.

4. Creutzfeldt W. Carcinoid tumors: development of our knowledge. World J Surg 1996; 20:|26-3|.

5. O'dowd G, Gosney JR. Absence of over expression of $\mathrm{p} 53$ protein by intestinal carcinoid tumors. I Pathol 1995; 175:403-4

6. Öberg K. Biological aspects of neuroendocrine gastro-enteropancreatic tumours. Digestion 1996; 57(Suppl 1):42-4.

7. Woods HF, Bax ND, Ainsworth I. Abdominal carcinoid tumors in Sheffield. Digestion 1990; 45(Suppl I): 17-22.

8. Newton JN, Swerdlow AJ, Santos Silva IM, Vessey MP, Grahame-Smith DG, Primatesta P et al. The epidemiology of carcinoid tumors in England and Scotland. Br J Cancer 1994; 70:939-42.

9. Neary PC, Redmond PH, Houghton T, Watson GR, Bouchier-Hayes D. Carcinoid disease: review of the literature. Dis Colon Rectum 1997; 40:349-62.

10. Zeitels J, Naunheim K, Kaplan El, Straus F. Carcinoid tumors: a 37 year experience. Arch Surg 1982; 117:732-7.

I I. Modlin IM, Sandor A. An analysis of 8305 cases of carcinoid tumors. Cancer 1997; 79:813-29.

12. Williams ED, Sandler M. The classification of carcinoid tumors. Lancet 1963; 1:238-9.

13. Faiss S, Scherübl H, Riecken EO, Wiedenmann B. The ultimate biochemical diagnosis of neuroendocrine gastro-enteropancreatic tumours. Digestion 1996; 57 (Suppl I): 48-9.

14. Taal BG, Hoefnagel CA, Valdés Olmos RA, Boot $\mathrm{H}$. Combined diagnostic imaging with | 3 ||-Metaiodobenzy|guanidine and | | |lnpentetreotide in carcinoid tumours. Eur | Cancer 1996; 32A: 1924-32.

15. Paulson EK, Mcdermott VG, Keogan MT, DE
Long DM, Frederick MG, Nelson RC. Carcinoid metastases to the liver: role of triple-phase helical CT. Radiology 1998; 206: 143-50.

16. Chayvialle JA. A comparison of imaging techniques for the localisation of gastroenteropancreatic neuroendocrine tumours. Digestion 1996; 57(Suppl I):54-6.

17. Yoshikane H, Tsukamoto Y, Niwa Y, Goto $\mathrm{H}$, Hase S, Mizutani K et al. Carcinoid tumors of the gastrointestinal tract: evaluation with encoscopic ultrasonography. Gastrointest Endosc 1993; 39:375-83.

18. Hokama A, Oshiro J, Kinjo F, Saito A. Utility of endoscopic ultrasonography in rectal carcinoid tumors. Am J Gastroenterol 1996; 91: 1289-90.

19. Anthony LB, Martin W, Delbeke D, Sandler M. Somatostatin receptor imaging: predictive and prognostic considerations. Digestion 1996; 57(Suppl 1):50-3

20. Modlin IM, Cornelius E, Lawton GP. Use of an isotopic somatostatin receptor probe to image gut endocrine tumors. Arch Surg 1995; 130:367-73.

21. Akeström G. Management of carcinoid tumors of the stomach, duodenum and pancreas. World J Surg 1996, 20:173-82.

22. Lehy T, Mignon M, Cadiot G, Elouaer-Blanc L, Ruszniewski P, Lewin MJ et al. Gastric endocrine cell behaviour in Zollinger-Ellison patients on long term potent antisecretory treatment. Gastroenterology 1989; 96: 1029-40.

23. Muller J, Kirchner T, Muller-Hermelink HK. Gastric endocrine cell hyperplasia and carcinoid tumors in atrophic gastritis type A. Am J Surg Pathol 1987; 1: 909-17.

24. Rindi G, Luineti $O$, Cornaggia M, Capella C, Solcia E. Three subtypes of gastric argyrophil carcinoid and the gastric neuroendocrine carcinoma: a clinicopathologic study. Gastroenterology 1993; 105:1264-6.

25. Caplin Me, Buscombe Jr, Hilson Aj, Jones Al, Watkinson Af, Burroughs AK. Carcinoid tumor. Lancet 1998; 352:799-805.

26. Takami H, Miyoshi H, Makuuchi H. Strip biopsy for gastrointestinal carcinoid tumor, J Surg Oncol 1998; 68:60-I

27. Modlin IM, Gilligan C), Lawton GP, Tang LH, West AB, Darr H. Gastric carcinoids: the Yale experience. Arch Surg 1995, 130:250-6.

28. Attanoos R, Williams GT. Epithelial and neuroendocrine tumors of the duodenum. Semin Diagn Pathol 199|, 8:|49-54

29. Nishimori I, Morita M, Sano S, Kino-Ohsaki J, Kohsaki T, Suenaga $\mathrm{K}$ et al. Endosonographyguided endoscopic resection of duodenal carcinoid tumor. Endoscopy 1997, 29:214-7.

30. Yoshikane H, Goto H, Niwa Y, Matsui M, Ohashi S, Suzuki Tl et al. Endoscopic resection of small duodenal carcinoid tumors with strip biopsy technique. Gastrointest Endosc $1998,6: 466-70$

31. Zimmer T, Stülzel U, Bäder M, Koppenhagen K, Hamm B, Buhr $\mathrm{H}$ et al. Endoscopic ultrasonography and somatostatin receptor scintigraphy in the preoperative localisation of insulinomas and gastrinomas. Gut 1996, 39:562-8.
32. Stinner B, Kisker $O$, Zielke A, Rothmund M. Surgical management for carcinoid tumors of small bowel, appendix, colon and rectum. World J Surg 1996; 20:183-8.

33. Memon MA, Nelson H. Gastrointestinal carcinoid tumors: current management strategies. Dis Colon Rectum 1997; 40:1 I0I-I3.

34. Makridis C, Öberg K, Juhlin C, Rastad J Johansson $H$, Lürelius LE et al. Surgical treatment of mid-gut carcinoid tumors. World J Surg 1990; 14:377-83.

35. Deans GT, Spence RA. Neoplastic lesions of the appendix. Br J Surg 1995; 82:299-306.

36. Wallace S, Ajani JA, Charnsangavej C, Du Brow R, Yang Dj, Chuang VP et al. Carcinoid tumors: imaging procedures and interventional radiology. World I Surg 1996: 20:147-56.

37. Bhutani MS. Curative endoscopic resection of a carcinoid tumor of the rectum. Am J Gastroenterol 1994; 89:645.

38. Naunheim KS, Zeitels J, Kaplan El, Sugimoto J. Shen KI, Lee $\mathrm{CH}$ et al. Rectal carcinoid tumors: treatment and prognosis. Surgery 1983; 94:670-6.

39. Koura AN, Giacco GG, Curley SA, Skibber $J M$, Feig BW, Ellis LM. Carcinoid tumors of the rectum: effect of size, histopathology, and surgical treatment on metastasis free survival. Cancer 1997; 79:1294-8.

40. Schindl M, Niederle B, Häfner M, Teleky B, Längle $F$, Kaserer $K$ et al. Stage-dependent therapy of rectal carcinoid tumors. World J Surg 1998; 22:628-34.

4I. Chen H, Hardacre JM, Uzar A, Cameron JI, Choti MA. Isolated liver metastases from neuroendocrine tumors: does resection prolong survival? J Am Coll Surg 1998; 187:88-92.

42. Frilling A, Malago M, Martin H, Broelsch CE. Use of somatostatin receptor scitigraphy to image extrahepatic metastases of neuroendocrine tumors. Surgery 1998:124:1000-4.

43. Campos FGCM, Silva JH, Habr-Gama A Rodrigues JG, Carneiro LA, Pinotti HW. Rectal carcinoid with hepatic metastasis: a case report. Rev Bras Coloproctol 1989; 9: I16-9.

44. Ahlman H, Westberg G, Wängberg B, Nilsson $O$, Tylén $U$, Scherstén $T$ et al. Treatment of liver metastases of carcinoid tumours. World Surg 1996; 20:196-202.

45. Bilchik AJ, Sarantou T, Foshag LJ, Giuliano AE, Ramming KP. Cryosurgical palliation of metastatic neuroendocrine tumors resistant to conventional therapy. Surgery 1997; 1 22: 1040-7

46. Dale PS, Souza JW, Brewer DA. Cryosurgical ablation of unresectable hepatic metastases. J Surg Oncol 1998; 68:242-5

47. Frilling $A$, Rogiers $X$, Knüfel WT, Broelsch CE Liver transplantation for metastatic carcinoid tumors. Digestion 1994; 55(Suppl 3): I04-6.

48. Anthuber M, Jauch KW, Briegel J, Groh J, Schildberg FW. Results of liver transplantation for gastroenteropancreatic tumor metastases. World J Surg 1996; 20:73-6.

49. Chakravarthy A, Abrams RA. Radiation therapy in the management of patients with malignant carcinoid tumors. Cancer 1995; $75: 1386-90$ 
Fernandes LC ETAL.

50. Bajetta E, Rimassa L, Carnaghi C, Seregni E, Ferrari L, Di Bartolomeo M et al. 5-Fluorouracil, dacarbazine, and epirubicin in the treatment of patients with neuroendocrine tumors. Cancer 1998; 83:372-8.

51. Nobin A, Lindblom A, Månsson B, Sundberg M. Interferon treatment in patients with malignant carcinoids. Acta Oncol 1989; 28:445-9.

52. Saltz L, Kemeny N, Schwartz G, Kelsen D. A phase II trial of alpha interferon and 5-fluorouracil in patients with advanced carcinoid and islet cell tumors. Cancer 1994; 74:958-61.

53. Kvols LK, Martin JK, Marsh HM, Moertel CG. Rapid reversal of carcinoid crisis with a somatostatin analogue. N Engl J Med 1985; 313: 1229

54. Saslow SB, O'brien MD, Camilleri M, Oke M, Homburger HA, Klee GG et al. Oc treotide inhibition of flushing and colonic motor disfunction in carcinoid syndrome. Am J Gastroenterol 1997; 92:2250-6.

55. Eriksson B, Janson ET, Bax ND, Mignon M, Morant R, Opolon P et al. The use of new somatostatin analogues, lanreotide and octastatin, in neuroendocrine gastro-intestinal tumours. Digestion 1996; 57(Suppl 1):77-80.
56. Arnold R, Frank M. Control of growth in neuroendocrine gastro-enteropancreatic tumours. Digestion 1996; 57(Suppl I):69-7I.

57. Bajetta E, Carnaghi C, Ferrari L, Spagnoli I, Mazzaferro $V$, Buzzoni R. The role of somatostatin analogues in the treatment of gastro-enteropancreatic endocrine tumours. Digestion 1996; 57(Suppl I):72-6.

58. Faiss S, Scherübl H, Riecken EO, Wiedenmann B. Interferon- $\mu$ versus somatostatin or the combination of both in metastatic neuroendocrine gut and pancreatic tumours. Digestion 1996; 57(Suppl I):84-5

59. McDermott WV. Hersle TW. Metastatic carcinoid to the liver treated by hepatic dearterialisation. Ann Surg 1974: 180:305-8.

60. Ahlman $\mathrm{H}$. The role of surgery in patients with advanced midgut carcinoid tumours. Digestion 1996; 57(Suppl 1):86-7

61. Drougas JG, Anthony LB, Blair TK, Lopez RR, Wright JK, Chapman WC et al. Hepatic artery chemoembolization for management of patients with advanced metastatic carcinoid tumors. Am । Surg 1998; 175:408-12
62. Godwin JD. Carcinoid tumors: an analysis of 2,837 cases. Cancer 1975; 36:560-9.

63. McDermott EW, Guduric B, Brennan MF. Prognostic variables in patients with gastrointestinal carcinoid tumors. Br J Surg 1994: 81:1007-9.

64. Waisberg J, Hamada M, Gonçalves JE, Messias M, Bromberg SH, Jatobá PP et al. Tumores carcinóides do trato gastrintestinal: análise de 21 casos. Arq Gastroenterol 1990; 27:53-61.

65.Aranha GV, Greenlee HB. Surgical management of carcinoid tumors of the gastrointestinal tract. Am J Surg 1980; 46: 429-35.

66. Sandor A, Modlin IM. A retrospective analysis of 1570 appendiceal carcinoids. Am J Gastroenterol 1998; 93:422-8.

67. Soga J. Carcinoids of the rectum: an evaluation of 127| reported cases. Jpn J Surg 1997: 27: $12-9$.

Artigo recebido: 15//2/2000 Aceito para publicação: 19/06/200 |

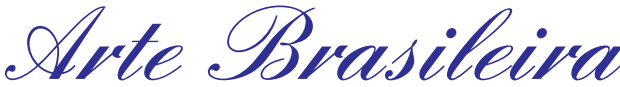

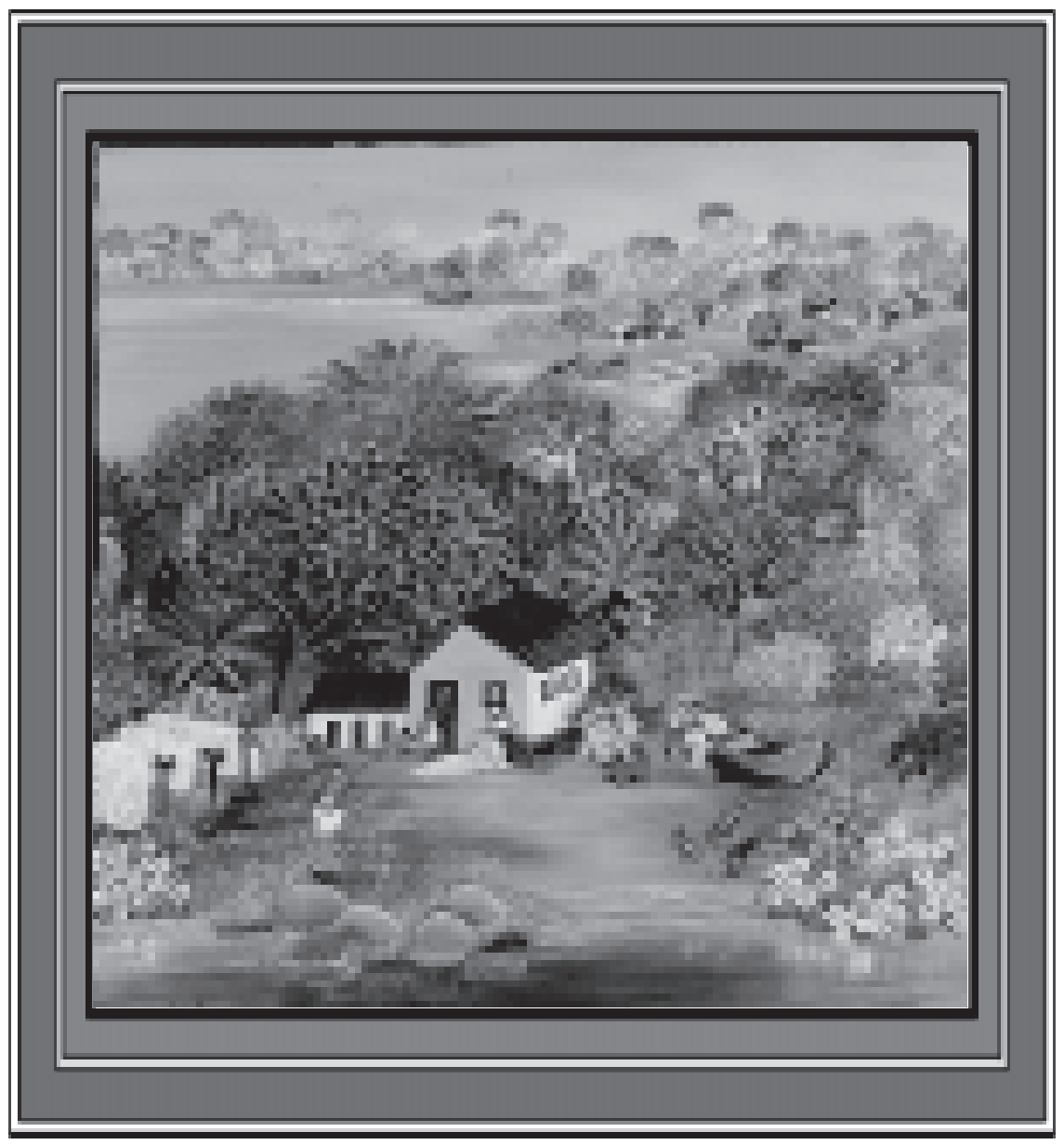

Lúcia Buccini - "Casa de Pescador" - Galeria Jacques Ardies - Tel.: (II) 3884-2916 